\title{
Projective Surfaces and Pre-Normalized Blaschke Immersions of Codimension Two
}

\author{
Atsushi Fujioka, Hitoshi Furuhata * and Takeshi Sasaki \\ (Communicated by Erdal ÖZÜSAĞLAM)
}

\begin{abstract}
We prove that any non-degenerate surface in the projective 3-space has a local lift as a minimal pre-normalized Blaschke immersion into the equicentroaffine 4 -space. Furthermore, an indefinite surface in the projective 3-space has a local lift as a pre-normalized Blaschke immersion into the equicentroaffine 4-space satisfying the Einstein condition if and only if the surface is projectively applicable to an affine sphere.
\end{abstract}

Keywords: projective surface; affine sphere; pre-normalized Blaschke immersion; centroaffine minimality; Einstein condition.

AMS Subject Classification (2010): Primary: 53A15; Secondary: 53A20, 53A07

\section{Introduction}

Differential geometry of surfaces in the real projective space $\mathbf{P}^{3}$ has a long history from the early twentieth century. We can find a lot of papers and books concerning about this topic in references in $[1,8,9]$.

In the previous paper [2], the authors studied centroaffine surfaces in the affine space $\mathbf{R}^{3}$ from the viewpoint of projective differential geometry by regarding centroaffine surfaces in $\mathbf{R}^{3}$ as surfaces in $\mathbf{P}^{3}$. In contrast to [2], in this article, we shall study projective surfaces in $\mathbf{P}^{3}$ from the viewpoint of equicentroaffine differential geometry of codimension two by regarding projective surfaces in $\mathbf{P}^{3}$ as surfaces in $\mathbf{R}^{4}$.

In 1993, Nomizu and Sasaki [6] gave a new approach by using the equicentroaffine geometry of surfaces in $\mathbf{R}^{4}$. A point of such geometry of codimension two is how to take transversal vector fields for a surface. One transversal vector field is the radial vector field, that is, the position vector field of a surface, and the other is chosen to be a pre-normalized Blaschke normal vector field, which was defined in [6]. See [4, 5, 11, 12] for other choices of transversal vector fields. Following [6], Furuhata [3] studied surfaces in $\mathbf{R}^{4}$ with vanishing shape operator, which can be considered from a viewpoint of a certain variation problem. We call them minimal prenormalized Blaschke surfaces in this article. It is a natural question to determine surfaces in $\mathbf{P}^{3}$ admitting local lifts as minimal pre-normalized Blaschke surfaces in $\mathbf{R}^{4}$. In this article, we give an answer to this question, which claims any surface has such a lift (Theorem 5.1).

It is an interesting problem to characterize a surface in $\mathbf{P}^{3}$ in terms of the property whether it has a certain special lift in $\mathbf{R}^{4}$ or not. Affine spheres are important objects not only in equiaffine differential geometry, but also in projective differential geometry. We show that a surface projectively applicable to an affine sphere is characterized to have a local lift such that the Ricci tensor field of the induced connection is constant multiple of the pre-normalized Blaschke metric (Theorem 6.1).

\section{Projective surfaces}

We denote by $\mathbf{P}^{n}$ the real projective space of dimension $n$. In this section, we shall review the surface theory in $\mathbf{P}^{3}$. See $[1,8,9]$ and references therein for more detail. 
A projective surface is an immersion from a 2-dimensional manifold $M$ into $\mathbf{P}^{3}$. If we take local coordinates $(x, y)$ on $M$, a projective surface $\underline{z}$ is given by a lift $z$ into $\mathbf{R}^{4} \backslash\{0\}$ :

$$
z(x, y)=\left(z^{1}(x, y), z^{2}(x, y), z^{3}(x, y), z^{4}(x, y)\right)
$$

where

$$
\underline{z}(x, y)=\left[z^{1}(x, y): z^{2}(x, y): z^{3}(x, y): z^{4}(x, y)\right]
$$

via homogeneous coordinates on $\mathbf{P}^{3}$. In the following, we assume that the vectors $z_{x y}, z_{x}, z_{y}, z$ are linearly independent at each point $(x, y)$, which is independent of the choice of the lift. Then $z_{x x}$ and $z_{y y}$ can be written as

$$
z_{x x}=l z_{x y}+a z_{x}+b z_{y}+p z, z_{y y}=m z_{x y}+c z_{x}+d z_{y}+q z
$$

for some functions $l, m, a, b, c, d, p, q$. Given a projective surface $\underline{z}$, we associate a surface in $\mathbf{R}^{3}$ by fixing inhomogeneous coordinates: for example, for the mapping $\underline{z}$ above and the inhomogeneous coordinates $[1: u: v: w]$, we have a surface in $\mathbf{R}^{3}$ as

$$
\left(z^{2}(x, y) / z^{1}(x, y), z^{3}(x, y) / z^{1}(x, y), z^{4}(x, y) / z^{1}(x, y)\right) .
$$

It is easy to see that the symmetric $(0,2)$-tensor $\psi$ defined by

$$
\psi=l d x^{2}+2 d x d y+m d y^{2}
$$

is conformal to the second fundamental form of the surface in $\mathbf{R}^{3}$. We call $\underline{z}$ an indefinite projective surface if $\psi$ is indefinite.

In the following, we assume that $\underline{z}$ is an indefinite projective surface. Then taking asymptotic line coordinates for the corresponding surface in $\mathbf{R}^{3}$, that is, taking coordinates so that $\ell=m=0$, and rescaling the lift, we may assume that a lift $z$ satisfies a system of the form

$$
z_{x x}=b z_{y}+p z, z_{y y}=c z_{x}+q z
$$

which is called a canonical system.

It is straightforward to see that the integrability condition for (2.1) is given by

$$
\left\{\begin{array}{l}
L_{y}=-2 b c_{x}-c b_{x}, M_{x}=-2 c b_{y}-b c_{y} \\
b M_{y}+2 M b_{y}+b_{y y y}=c L_{x}+2 L c_{x}+c_{x x x}
\end{array}\right.
$$

where

$$
L=-b_{y}-2 p, M=-c_{x}-2 q .
$$

Definition 2.1. Let $\underline{z}$ and $\underline{w}$ be indefinite projective surfaces and choose a lift $z$ of $\underline{z}$ satisfying (2.1). We say that $\underline{w}$ is projectively applicable to $\underline{z}$ if $\underline{w}$ has a lift $w$ satisfying a canonical system with the same $b$ and $c$ in (2.1).

By use of transformation formulas for $b$ and $c$, it is easy to see that the above definition is independent of choice of $z$. In other references, it might be assumed in addition that $\underline{w}$ is not projectively equivalent to $\underline{z}$.

\section{Affine spheres}

We first recall a formulation of the theory of affine hypersurfaces and then we define a notion of affine spheres in $\mathbf{P}^{3}$. Such surfaces are found to form an important class of projective surfaces. See $[7,10]$ for more detail.

For an immersion $F$ from an $n$-dimensional manifold $M$ into the affine space $\mathbf{R}^{n+1}$ with the standard flat connection $D$, we choose a transversal vector field $\xi$ along $F$. Then, the Gauss-Weingarten formulas for the immersion $F$ are given by

$$
\left\{\begin{array}{l}
D_{X} F_{*} Y=F_{*} \nabla_{X} Y+h(X, Y) \xi, \\
D_{X} \xi=-F_{*} S X+\tau(X) \xi
\end{array} \quad(X, Y \in \mathfrak{X}(M)),\right.
$$

where $\mathfrak{X}(M)$ is the set of all vector fields on $M$. Then $\nabla, h, S$ and $\tau$ define a torsion-free affine connection, a symmetric $(0,2)$-tensor, a $(1,1)$-tensor and a 1 -form on $M$, respectively, which we call the induced connection, the 
affine fundamental form, the affine shape operator and the transversal connection form, respectively. We fix a volume form $\omega$ on $\mathbf{R}^{n+1}$ which is parallel with respect to $D$ and define a volume form $\theta$ on $M$ by

$$
\theta\left(X_{1}, \ldots, X_{n}\right)=\omega\left(F_{*} X_{1}, \ldots, F_{*} X_{n}, \xi\right)
$$

for $X_{1}, \ldots, X_{n} \in \mathfrak{X}(M)$, called the volume form induced by $F$ and $\xi$. Then we have

$$
\nabla_{X} \theta=\tau(X) \theta \quad(X \in \mathfrak{X}(M)) .
$$

We call $F$ to be non-degenerate if $h$ is non-degenerate; this property is independent of the choice of $\xi$. Then we can find a transversal vector field $\xi$ such that $\tau=0$. We call the immersion $F$ with such a vector field $\xi$ an equiaffine immersion. Moreover, we can find a unique transversal vector field $\xi$ up to sign such that $\tau=0$ and $\theta$ is equal to the volume form with respect to $h$. Then, we call $F$ with $\xi$ a Blaschke immersion, and $\xi$ the Blaschke normal vector field of $F$.

A Blaschke immersion is called an affine sphere if the affine shape operator $S$ is a scalar operator. An affine sphere is said to be proper or improper if $S$ is nonzero or zero, respectively.

Definition 3.1. A projective surface in $\mathbf{P}^{3}$ is called an affine sphere if it is locally an affine sphere in some affine chart $\mathbf{R}^{3}$ in $\mathbf{P}^{3}$.

If we use the canonical system (2.1), the integrability condition for indefinite affine spheres in $\mathbf{P}^{3}$ can be stated as follows.

Lemma 3.1. Let $z$ be a lift of an indefinite projective surface satisfying (2.1) and

$$
z=\left(e^{\frac{1}{2} \varphi}, e^{\frac{1}{2} \varphi} F\right)
$$

for an $\mathbf{R}$-valued function $\varphi$ and a surface $F$ in $\mathbf{R}^{3}$. Then $F$ is an affine sphere if and only if there exists some $k \in \mathbf{R}$ such that

$$
\begin{gathered}
b_{y}=b \varphi_{y}, c_{x}=c \varphi_{x}, \\
\varphi_{x y}=b c+k e^{-\varphi} .
\end{gathered}
$$

Moreover, $F$ is a proper affine sphere if $k \neq 0$ and an improper affine sphere if $k=0$.

Proof. From (2.1) and (3.1), we have

$$
\begin{gathered}
p=\frac{1}{2} \varphi_{x x}+\frac{1}{4} \varphi_{x}^{2}-\frac{1}{2} b \varphi_{y}, q=\frac{1}{2} \varphi_{y y}+\frac{1}{4} \varphi_{y}^{2}-\frac{1}{2} c \varphi_{x}, \\
F_{x x}=-\varphi_{x} F_{x}+b F_{y}, F_{y y}=c F_{x}-\varphi_{y} F_{y} .
\end{gathered}
$$

Note that $\omega\left(F_{x}, F_{y}, F_{x y}\right) \neq 0$, since $\underline{z}$ is indefinite. If we put

$$
\xi=\lambda F_{x y}, \lambda^{2}= \pm \frac{1}{\omega\left(F_{x}, F_{y}, F_{x y}\right)},
$$

then, from (3.5), it is straightforward to see that

$$
(\log \lambda)_{x}=\varphi_{x},(\log \lambda)_{y}=\varphi_{y}
$$

and hence,

$$
\left(\begin{array}{c}
\xi_{x} \\
\xi_{y}
\end{array}\right)=\lambda\left(\begin{array}{cc}
-\varphi_{x y}+b c & -b \varphi_{y}+b_{y} \\
-c \varphi_{x}+c_{x} & -\varphi_{x y}+b c
\end{array}\right)\left(\begin{array}{c}
F_{x} \\
F_{y}
\end{array}\right),
$$

which shows that $F$ is an equiaffine immersion with $\xi$. Moreover, it is easy to see that $\xi$ is a Blaschke normal vector field.

Now we assume that $F$ is an affine sphere. Then from (3.6), we have (3.2) which can be solved as

$$
b=f(x) e^{\varphi}, c=g(y) e^{\varphi}
$$

for some functions $f$ and $g$ of one variable. Then from (2.2), (2.3), (3.4) and (3.7), we have

$$
\varphi_{x x y}+\varphi_{x} \varphi_{x y}=\left(3 f g \varphi_{x}+f^{\prime} g\right) e^{2 \varphi}, \varphi_{x y y}+\varphi_{y} \varphi_{x y}=\left(3 f g \varphi_{y}+f g^{\prime}\right) e^{2 \varphi},
$$

which are equivalent to

$$
\left(\varphi_{x y} e^{\varphi}-f g e^{3 \varphi}\right)_{x}=0,\left(\varphi_{x y} e^{\varphi}-f g e^{3 \varphi}\right)_{y}=0 .
$$

Hence there exists some $k \in \mathbf{R}$ such that

$$
\varphi_{x y} e^{\varphi}-f g e^{3 \varphi}=k,
$$

which is equivalent to (3.3) from (3.7). From (3.3) and (3.6), $F$ is proper if and only if $k \neq 0$. 


\section{Centroaffine immersions of codimension two}

Following [6], we shall explain the notion of centroaffine immersions of codimension two.

We denote by $\eta$ the radial vector field on $\mathbf{R}^{n+2}$. An immersion $F$ from an $n$-dimensional manifold $M$ into $\mathbf{R}^{n+2}$ is called a centroaffine immersion of codimension two if $\eta \circ F$ is a transversal vector field along $F$ and there exists a vector field $\xi$ along $F$ which is transversal to the direct sum of the vector space spanned by $(\eta \circ F)(x)$ and $F_{*} T_{x} M$ at each point $x \in M$. We denote by $D$ the standard flat connection on $\mathbf{R}^{n+2}$. If $f$ is a centroaffine immersion of codimension two, we have the following equations:

$$
\left\{\begin{array}{l}
D_{X} \eta=F_{*} X \\
D_{X} F_{*} Y=F_{*} \nabla_{X} Y+h(X, Y) \xi+T(X, Y) \eta, \\
D_{X} \xi=-F_{*} S X+\tau(X) \xi+\rho(X) \eta
\end{array}\right.
$$

for $X, Y \in \mathfrak{X}(M)$. Then $\nabla$ defines a torsion-free affine connection, and both $h$ and $T$ symmetric $(0,2)$-tensors on $M$. Moreover, $S$ defines a (1,1)-tensor, and both $\tau$ and $\rho$ are 1-forms on $M$. We fix a volume form $\omega$ on $\mathbf{R}^{n+2}$ which is parallel with respect to $D$ and define a volume form $\theta$ on $M$ by

$$
\theta\left(X_{1}, \ldots, X_{n}\right)=\omega\left(F_{*} X_{1}, \ldots, F_{*} X_{n}, \xi, \eta\right)
$$

for $X_{1}, \ldots, X_{n} \in \mathfrak{X}(M)$. Then we have

$$
\nabla_{X} \theta=\tau(X) \theta \quad(X \in \mathfrak{X}(M)) .
$$

The following is the fundamental result concerning about reduction of codimension of centroaffine immersions of codimension two.

Proposition 4.1 ([6]). Let $F$ be a centroaffine immersion of codimension two. In the case that rank $h \geq 2$, the image of $F$ is contained in some affine hyperplane if and only if $T=\lambda h$ for some function $\lambda$. If $h=0$ and $n \geq 2$, then the image of $F$ is contained in some affine hyperplane which goes through 0.

If $F$ is a centroaffine immersion of codimension two such that $h$ is non-degenerate, we call $F$ to be nondegenerate; this is independent of the choice of $\xi$. Let $F$ be a non-degenerate centroaffine immersion of codimension two. Then we can find a transversal vector field $\xi$ such that $\tau=0$. We call $F$ with such $\xi$ an equiaffine immersion. We can also find a transversal vector field $\xi$ determined $\bmod \eta$ up to sign such that $\tau=0$ and $\theta$ is equal to the volume form with respect to $h$. We call $F$ with such $\xi$ a Blaschke immersion. Moreover, we can find a unique transversal vector field $\xi$ up to sign such that it satisfies all the above conditions with the equation

$$
\operatorname{tr}_{h}((X, Y) \mapsto T(X, Y)+h(S X, Y))=0 .
$$

We call $F$ with such $\xi$ a pre-normalized Blaschke immersion, and $\xi$ the pre-normalized Blaschke normal vector field of F.

Definition 4.1 ([3]). A pre-normalized Blaschke immersion $F$ is called to be centroaffine minimal, or minimal for short, if it is extremal for the integral of the volume form $\theta$ among any variation in the pre-normalized Blaschke normal direction, which is equivalent to the condition $\operatorname{tr} S=0$.

Example 4.1. A curve in $\mathbf{P}^{2}$ is a map from an interval into $\mathbf{P}^{2}$. If we use homogeneous coordinates on $\mathbf{P}^{2}$, a curve in $\mathbf{P}^{2}$ is given by a lift $z$ into $\mathbf{R}^{3} \backslash\{0\}$ :

$$
z(t)=\left(z_{1}(t), z_{2}(t), z_{3}(t)\right) .
$$

In the following, we assume that the vectors $z^{\prime \prime}, z^{\prime}, z$ are linearly independent at each point $t$. Then $z_{1}, z_{2}, z_{3}$ can be given by linearly independent solutions of a third-order linear differential equation:

$$
z^{\prime \prime \prime}+p_{1} z^{\prime \prime}+p_{2} z^{\prime}+p_{3} z=0 .
$$

By the assumption, $z$ is a centroaffine immersion of codimension two with a transversal vector field $\xi=z^{\prime \prime}$. From (4.1), we have

$$
\nabla_{\frac{d}{d t}} \frac{d}{d t}=0, h\left(\frac{d}{d t}, \frac{d}{d t}\right)=1, T\left(\frac{d}{d t}, \frac{d}{d t}\right)=0 .
$$


In particular, if we denote by $\omega_{h}$ the volume form with respect to $h$, we have

$$
\omega_{h}\left(\frac{d}{d t}\right)=1
$$

From (4.1) and (4.3), we have

$$
S\left(\frac{d}{d t}\right)=p_{2}, \tau\left(\frac{d}{d t}\right)=-p_{1}, \rho\left(\frac{d}{d t}\right)=-p_{3}
$$

In particular, $z$ is an equiaffine immersion with $\xi$ if and only if $p_{1}=0$. The volume form $\theta$ is given by

$$
\theta\left(\frac{d}{d t}\right)=\omega\left(z^{\prime}, \xi, \eta\right)=\omega\left(z, z^{\prime}, z^{\prime \prime}\right)
$$

From (4.5) and (4.7), $z$ is a Blaschke immersion with $\xi$ if and only if

$$
p_{1}=0, \omega\left(z, z^{\prime}, z^{\prime \prime}\right)=1 .
$$

Moreover, from (4.4) and (4.6), we have

$$
T\left(\frac{d}{d t}, \frac{d}{d t}\right)+h\left(S\left(\frac{d}{d t}\right), \frac{d}{d t}\right)=p_{2}
$$

so that

$$
\operatorname{tr}_{h}\{(X, Y) \mapsto T(X, Y)+h(S X, Y)\}=p_{2} .
$$

Hence $z$ is a pre-normalized Blaschke immersion with $\xi$ if and only if $z$ is given by a Laguerre-Forsyth canonical form:

$$
z^{\prime \prime \prime}+p_{3} z=0
$$

with normalization

$$
\omega\left(z, z^{\prime}, z^{\prime \prime}\right)=1
$$

\section{Pre-normalized lifts and centroaffine minimality}

In this section, we shall consider lifts of indefinite projective surfaces in $\mathbf{P}^{3}$ as pre-normalized Blaschke immersions into $\mathbf{R}^{4}$. For an arbitrary chosen $\mathbf{R}$-valued function $\varphi$, we define a lift $w$ of $\underline{z}$ by $w=e^{-\frac{1}{2} \varphi} z$, where $z$ is a lift of $\underline{z}$ satisfying the canonical system (2.1). Then we have

$$
\left(w, w_{x}, w_{y}, w_{x y}\right)
$$

It follows that the vectors $w_{x y}, w_{x}, w_{y}, w$ are linearly independent at each point $(x, y)$ since we assume that the vectors $z_{x y}, z_{x}, z_{y}, z$ are linearly independent at each point $(x, y)$. For some scalar functions $\lambda$ and $\mu$, where $\lambda$ is assumed never to vanish, we define a transversal vector field $\xi$ given by

$$
\xi=\lambda w_{x y}+\mu w .
$$

Then, $w$ is a centroaffine immersion of codimension two with the transversal vector field $\xi$. We determine these functions $\lambda$ and $\mu$ so that the immersion $w$ with $\xi$ turns out to be a pre-normalized Blaschke immersion in the following. A direct computation shows that

$$
w_{x x}=-\varphi_{x} w_{x}+b w_{y}+\tilde{p} w, w_{y y}=c w_{x}-\varphi_{y} w_{y}+\tilde{q} w
$$


where

$$
\tilde{p}=-\frac{1}{2} \varphi_{x x}-\frac{1}{4} \varphi_{x}^{2}+\frac{1}{2} b \varphi_{y}+p, \tilde{q}=-\frac{1}{2} \varphi_{y y}-\frac{1}{4} \varphi_{y}^{2}+\frac{1}{2} c \varphi_{x}+q .
$$

Moreover, we have

$$
\left\{\begin{aligned}
\xi_{x} & =\left\{\lambda\left(-\varphi_{x y}+b c\right)+\mu\right\} w_{x}+\lambda\left(-b \varphi_{y}+b_{y}+\tilde{p}\right) w_{y} \\
& +\left(-\varphi_{x}+\frac{\lambda_{x}}{\lambda}\right) \xi+\left\{\mu \varphi_{x}+\lambda\left(\tilde{p}_{y}+b \tilde{q}\right)-\frac{\lambda_{x}}{\lambda} \mu+\mu_{x}\right\} w, \\
\xi_{y} & =\lambda\left(-c \varphi_{x}+c_{x}+\tilde{q}\right) w_{x}+\left\{\lambda\left(-\varphi_{x y}+b c\right)+\mu\right\} w_{y} \\
& +\left(-\varphi_{y}+\frac{\lambda_{y}}{\lambda}\right) \xi+\left\{\mu \varphi_{y}+\lambda\left(\tilde{q}_{x}+c \tilde{p}\right)-\frac{\lambda_{y}}{\lambda} \mu+\mu_{y}\right\} w .
\end{aligned}\right.
$$

From (4.1), (5.2), (5.3) and (5.4), we have the following:

$$
\begin{gathered}
\left\{\begin{array}{l}
\nabla_{\frac{\partial}{\partial x}} \frac{\partial}{\partial x}=-\varphi_{x} \frac{\partial}{\partial x}+b \frac{\partial}{\partial y}, \nabla_{\frac{\partial}{\partial y}} \frac{\partial}{\partial y}=c \frac{\partial}{\partial x}-\varphi_{y} \frac{\partial}{\partial y}, \\
\nabla_{\frac{\partial}{\partial x}} \frac{\partial}{\partial y}=\nabla_{\frac{\partial}{\partial y}} \frac{\partial}{\partial x}=0
\end{array}\right. \\
h\left(\frac{\partial}{\partial x}, \frac{\partial}{\partial x}\right)=h\left(\frac{\partial}{\partial y}, \frac{\partial}{\partial y}\right)=0, h\left(\frac{\partial}{\partial x}, \frac{\partial}{\partial y}\right)=\frac{1}{\lambda}, \\
T\left(\frac{\partial}{\partial x}, \frac{\partial}{\partial x}\right)=\tilde{p}, T\left(\frac{\partial}{\partial x}, \frac{\partial}{\partial y}\right)=-\frac{\mu}{\lambda}, T\left(\frac{\partial}{\partial y}, \frac{\partial}{\partial y}\right)=\tilde{q}, \\
S\left(\frac{\partial}{\partial y}\right)=\lambda\left(c \varphi_{x}-c_{x}-\tilde{q}\right) \frac{\partial}{\partial x}+\left\{\lambda\left(\varphi_{x y}-b c\right)-\mu\right\} \frac{\partial}{\partial y}, \\
\tau\left(\frac{\partial}{\partial x}\right)=\left\{\lambda\left(\varphi_{x y}-b c\right)-\mu\right\} \frac{\partial}{\partial x}+\lambda\left(b \varphi_{y}-b_{y}-\tilde{p}\right) \frac{\partial}{\partial y},
\end{gathered}
$$

Since $z$ satisfies (2.1), we have $\omega\left(z_{x}, z_{y}, z_{x y}, z\right)$ is a nonzero constant, and denote it by $C_{0}$. By (5.1), we have

$$
\begin{aligned}
\theta\left(\frac{\partial}{\partial x}, \frac{\partial}{\partial y}\right) & =\omega\left(w_{x}, w_{y}, \xi, w\right) \\
& =\lambda \omega\left(w_{x}, w_{y}, w_{x y}, w\right) \\
& =\lambda e^{-2 \varphi} \omega\left(z_{x}, z_{y}, z_{x y}, z\right) \\
& =C_{0} \lambda e^{-2 \varphi} .
\end{aligned}
$$

Lemma 5.1. Let $z$ be a lift of an indefinite projective surface in $\mathbf{P}^{3}$ satisfying (2.1). Set a centroaffine immersion $w=e^{-\frac{1}{2} \varphi} z$ of codimension two, and $\xi$ as in (5.2). Then $\xi$ is a pre-normalized Blaschke normal vector field of $w$ if and only if

$$
(\lambda, \mu)=C\left(e^{\varphi}, \frac{1}{2}\left(\varphi_{x y}-b c\right) e^{\varphi}\right)
$$

where $C$ is a nonzero constant $\pm\left|C_{0}\right|^{-1 / 2}$ as in (5.10).

Proof. From (5.9), $w$ with $\xi$ is an equiaffine immersion if and only if

$$
\lambda=C e^{\varphi}
$$

for some $C \in \mathbf{R} \backslash\{0\}$. From (5.6) and (5.10), $w$ with $\xi$ is a Blaschke immersion if and only if $C= \pm\left|C_{0}\right|^{-1 / 2}$ in (5.12).

From (5.6), (5.7) and (5.8), we have

$$
T\left(\frac{\partial}{\partial x}, \frac{\partial}{\partial y}\right)+h\left(S\left(\frac{\partial}{\partial x}\right), \frac{\partial}{\partial y}\right)=\varphi_{x y}-b c-2 \frac{\mu}{\lambda},
$$

which shows that it is a pre-normalized Blaschke immersion if and only if (5.11) holds. 
Let $\underline{z}$ be an indefinite projective surface in $\mathbf{P}^{3}, z$ a lift satisfying (2.1) and $w=e^{-\frac{1}{2} \varphi} z$ a lift as a pre-normalized Blaschke immersion with $\xi$ given by (5.2) with (5.11). Then, from (5.8) and (5.11), we have

$$
\operatorname{tr} S=C\left(\varphi_{x y}-b c\right) e^{\varphi} .
$$

Theorem 5.1. Any indefinite projective surface in $\mathbf{P}^{3}$ has a local lift as a minimal pre-normalized Blaschke immersion of codimension two.

Proof. From (5.13) it is enough to consider the equation

$$
\varphi_{x y}=b c
$$

which can be solved locally.

The same holds for a definite projective surface; refer to Corollary A.1.

Remark 5.1. In Theorem 5.1, let $w$ be a local lift as a minimal pre-normalized Blaschke immersion of codimension two. Then a local lift $f(x) g(y) w$ for functions $f$ and $g$ of one variable has also the same property.

\section{Pre-normalized lifts and Einstein condition}

In this section, we shall study properties of a lift of a surface projectively applicable to an affine sphere.

Let $z$ be a lift of an indefinite projective surface satisfying (2.1) and $w=e^{-\frac{1}{2} \varphi} z$ a lift as a pre-normalized Blaschke immersion with $\xi$ given by (5.2) with (5.11). If we denote by $R$ and Ric the curvature tensor and the Ricci tensor of $\nabla$ for $w$, respectively, from (5.5) we have

$$
\left\{\begin{array}{l}
R\left(\frac{\partial}{\partial x}, \frac{\partial}{\partial y}\right) \frac{\partial}{\partial x}=\left(\varphi_{x y}-b c\right) \frac{\partial}{\partial x}+\left(b \varphi_{y}-b_{y}\right) \frac{\partial}{\partial y} \\
R\left(\frac{\partial}{\partial y}, \frac{\partial}{\partial x}\right) \frac{\partial}{\partial y}=\left(c \varphi_{x}-c_{x}\right) \frac{\partial}{\partial x}+\left(\varphi_{x y}-b c\right) \frac{\partial}{\partial y}
\end{array}\right.
$$

so that

$$
\left\{\begin{array}{l}
\operatorname{Ric}\left(\frac{\partial}{\partial x}, \frac{\partial}{\partial x}\right)=-b \varphi_{y}+b_{y}, \operatorname{Ric}\left(\frac{\partial}{\partial y}, \frac{\partial}{\partial y}\right)=-c \varphi_{x}+c_{x} \\
\operatorname{Ric}\left(\frac{\partial}{\partial x}, \frac{\partial}{\partial y}\right)=\varphi_{x y}-b c
\end{array}\right.
$$

We call the condition

$$
\text { Ric }=\alpha h
$$

for a constant $\alpha \in \mathbf{R}$ the Einstein condition for a pre-normalized Blaschke immersion.

Theorem 6.1. An indefinite projective surface in $\mathbf{P}^{3}$ has a lift as a pre-normalized Blaschke immersion into $\mathbf{R}^{4}$ satisfying the Einstein condition if and only if the surface is projectively applicable to an affine sphere.

Proof. Let $z$ be a lift of an indefinite projective surface $z$ satisfying (2.1) and $w=e^{-\frac{1}{2} \varphi} z$ a lift as a pre-normalized Blaschke immersion with $\xi$ given by (5.2) with (5.11). If we put $\alpha=C k$, from (5.6), (5.11) and (6.1), the condition (6.2) is equivalent to (3.3) and (3.2), which implies that $\underline{z}$ is projectively applicable to an affine sphere given by $\tilde{z}=\left(e^{\frac{1}{2} \varphi}, e^{\frac{1}{2} \varphi} F\right)$.

\section{A. Equicentroaffine geometry of immersions of codimension two}

We now give equicentroaffine geometric properties of pre-normalized Blaschke immersions of an $n$ dimensional manifold into $\mathbf{R}^{n+2}$, though the basic is already stated in Section 4 . It is not necessary to assume $h$ is indefinite in this section.

At first, we note the formulas below in a general setting. 
Remark A.1. Let $\nabla$ be an affine connection of torsion free, and $h$ a pseudo-Riemannian metric on an $n$ dimensional manifold $M$. Let $\nabla^{*}$ be the dual connection of $\nabla$ with respect to $h$, which is by definition given as

$$
X h(Y, Z)=h\left(\nabla_{X} Y, Z\right)+h\left(Y, \nabla_{X}^{*} Z\right)
$$

for any vector fields $X, Y, Z \in \mathfrak{X}(M)$. Then,

$$
\operatorname{div}^{\nabla} \operatorname{grad}_{h} \psi=\operatorname{tr}_{h} \operatorname{Hess}^{\nabla^{*}} \psi
$$

holds for any function $\psi$ on $M$, where $\operatorname{div}^{\nabla}$ denotes the divergence relative to $\nabla$, $\operatorname{grad}_{h}$ the gradient relative to $h$, and Hess $\nabla^{*}$ the Hessian relative to $\nabla^{*}$. In fact, taking temporarily an orthonormal frame $X_{i} \in \mathfrak{X}(M)$ with respect to $h$ such that $h\left(X_{i}, X_{j}\right)=\varepsilon_{i} \delta_{i j}, \varepsilon_{i}= \pm 1$, we have

$$
\begin{aligned}
\text { LHS of (A.1) } & =\operatorname{tr}\left\{X \mapsto \nabla_{X} \operatorname{grad}_{h} \psi\right\} \\
& =\sum_{j} \varepsilon_{j} h\left(X_{j}, \nabla_{X_{j}} \operatorname{grad}_{h} \psi\right) \\
& =\sum_{j} \varepsilon_{j}\left\{X_{j} h\left(X_{j}, \operatorname{grad}_{h} \psi\right)-h\left(\nabla_{X_{j}}^{*} X_{j}, \operatorname{grad}_{h} \psi\right)\right\} \\
& =\sum_{j} \varepsilon_{j}\left\{X_{j}\left(X_{j} \psi\right)-\nabla_{X_{j}}^{*} X_{j} \psi\right\}=\text { RHS of (A.1). }
\end{aligned}
$$

If the volume form of $h$ is parallel with respect to $\nabla$, then

$$
\triangle_{h} \psi=\operatorname{div}^{\nabla^{h}} \operatorname{grad}_{h} \psi=\operatorname{div}^{\nabla} \operatorname{grad}_{h} \psi
$$

where $\nabla^{h}$ is the Levi-Civita connection of $h$, and $\triangle_{h}$ is the Laplacian with respect to $h$.

Let $D$ be the standard flat connection of $\mathbf{R}^{n+2}$, and $\omega$ a $D$-parallel volume form on $\mathbf{R}^{n+2}$. Let $F: M \rightarrow \mathbf{R}^{n+2}$ be a pre-normalized Blaschke immersion of an $n$-dimensional manifold $M$ with normal $\xi$, and $\nabla, h, T, S, \rho$ as in the formulas (4.1). For a function $\psi$ on $M$, we set $\widetilde{F}=e^{\psi} F$ and take its pre-normalized Blaschke normal vector field $\widetilde{\xi}$. Define $\widetilde{\nabla}, \widetilde{h}, \widetilde{T}, \widetilde{S}$ and $\widetilde{\rho}$ by

$$
\begin{aligned}
& D_{X} \widetilde{F}_{*} Y=\widetilde{F}_{*} \widetilde{\nabla}_{X} Y+\widetilde{h}(X, Y) \widetilde{\xi}+\widetilde{T}(X, Y) \widetilde{\eta}, \\
& D_{X} \widetilde{\xi}=-\widetilde{F}_{*} \widetilde{S} X+\widetilde{\rho}(X) \widetilde{\eta}
\end{aligned}
$$

for $X, Y \in \mathfrak{X}(M)$, where $\widetilde{\eta}$ is the radial vector field for $\widetilde{F}$.

Lemma A.1. For pre-normalized Blaschke immersions $F, \widetilde{F}=e^{\psi} F: M \rightarrow \mathbf{R}^{n+2}$, the following equations hold.

$$
\begin{aligned}
& \widetilde{\nabla}_{X} Y=\nabla_{X} Y+(Y \psi) X+(X \psi) Y-h(X, Y) \operatorname{grad}_{h} \psi, \\
& \widetilde{h}=e^{2 \psi} h, \\
& \widetilde{T}=T+\operatorname{Hess}^{\nabla} \psi-d \psi \otimes d \psi+\frac{1}{2}\left|\operatorname{grad}_{h} \psi\right|_{h}^{2} h, \\
& \widetilde{S}=e^{-2 \psi}\left\{S-\frac{1}{2}\left|\operatorname{grad}_{h} \psi\right|_{h}^{2} \mathrm{Id}+\operatorname{grad}_{h} \psi \otimes d \psi-\nabla \operatorname{grad}_{h} \psi\right\} .
\end{aligned}
$$

Proof. We express the pre-normalized Blaschke normal vector filed $\widetilde{\xi}$ by

$$
u \widetilde{\xi}=F_{*} U+\xi+a \eta,
$$


where $U$ is a vector field, $u, a$ are functions and $u$ is positive. Since $\widetilde{F}_{*} Y=e^{\psi} F_{*} Y+\left(Y e^{\psi}\right) \eta$, we have

$$
\begin{aligned}
& D_{X} \widetilde{F}_{*} Y= F_{*}\left\{e^{\psi} \nabla_{X} Y+\left(Y e^{\psi}\right) X+\left(X e^{\psi}\right) Y\right\} \\
&+e^{\psi} h(X, Y) \xi+\left\{e^{\psi} T(X, Y)+X\left(Y e^{\psi}\right)\right\} \eta, \\
& \widetilde{F}_{*} \widetilde{\nabla}_{X} Y+\widetilde{h}(X, Y) \widetilde{\xi}+\widetilde{T}(X, Y) \widetilde{\eta} \\
&=F_{*}\left\{e^{\psi} \widetilde{\nabla}_{X} Y+u^{-1} \widetilde{h}(X, Y) U\right\}+u^{-1} \widetilde{h}(X, Y) \xi \\
&+\left\{\widetilde{\nabla}_{X} Y e^{\psi}+u^{-1} a \widetilde{h}(X, Y)+e^{\psi} \widetilde{T}(X, Y)\right\} \eta, \\
& D_{X} \widetilde{\xi}= F_{*}\left\{-u^{-1} S X+u^{-1} a X+\left(X u^{-1}\right) U+u^{-1} \nabla_{X} U\right\} \\
&+\left\{X u^{-1}+u^{-1} h(X, U)\right\} \xi \\
&+\left\{u^{-1} \rho(X)+X\left(u^{-1} a\right)+u^{-1} T(X, U)\right\} \eta, \\
&-\widetilde{F}_{*} \widetilde{S} X+\widetilde{\rho}(X) \widetilde{\eta} \\
&=F_{*}\left\{-e^{\psi} \widetilde{S} X\right\}+\left\{e^{\psi} \widetilde{\rho}(X)-(\widetilde{S} X) e^{\psi}\right\} \eta .
\end{aligned}
$$

(Step 1) Comparing the $\xi$-components of (A.7) and (A.8), we have $\widetilde{h}=u e^{\psi} h$, and hence

$$
\left|\operatorname{det}\left(\widetilde{h}\left(X_{i}, X_{j}\right)\right)\right|^{1 / 2}=\left(u e^{\psi}\right)^{n / 2}\left|\operatorname{det}\left(h\left(X_{i}, X_{j}\right)\right)\right|^{1 / 2}
$$

for $X_{j} \in \mathfrak{X}(M)$. We calculate

$$
\begin{aligned}
& \omega\left(\widetilde{F}_{*} X_{1}, \ldots, \widetilde{F}_{*} X_{n}, \widetilde{\xi}, \widetilde{\eta}\right) \\
= & \omega\left(e^{\psi} F_{*} X_{1}, \ldots, e^{\psi} F_{*} X_{n}, u^{-1} \xi, e^{\psi} \eta\right) \\
= & e^{(n+1) \psi} u^{-1} \omega\left(F_{*} X_{1}, \ldots, F_{*} X_{n}, \xi, \eta\right) \\
= & e^{(n+1) \psi} u^{-1}\left|\operatorname{det}\left(h\left(X_{i}, X_{j}\right)\right)\right|^{1 / 2},
\end{aligned}
$$

from which (A.11) implies that

$$
u=e^{\psi}
$$

and hence (A.4).

(Step 2) Comparing the $\xi$-components of (A.9) and (A.10), we have

$$
U=\operatorname{grad}_{h} \psi
$$

by (A.12). Comparing $F_{*}$-components of (A.7) and (A.8), we get (A.3) from (A.4), (A.12) and (A.13). (Step 3) In a similar fashion, comparing the $\eta$-components of (A.7) and (A.8) implies

$$
\begin{aligned}
& \widetilde{T}(X, Y) \\
& =T(X, Y)+\operatorname{Hess}^{\nabla} \psi(X, Y)-(X \psi)(Y \psi) \\
& \quad+h(X, Y)\left\{\left|\operatorname{grad}_{h} \psi\right|_{h}^{2}-a\right\},
\end{aligned}
$$

and comparing the $F_{*}$-components of (A.9) and (A.10) implies

$$
\widetilde{S} X=e^{-2 \psi}\left\{S X-a X+(X \psi) \operatorname{grad}_{h} \psi-\nabla_{X} \operatorname{grad}_{h} \psi\right\},
$$

from which

$$
\begin{aligned}
& \operatorname{tr}_{\tilde{h}} \widetilde{T}+\operatorname{tr} \widetilde{S} \\
& =e^{-2 \psi}\left\{\operatorname{tr}_{h} T+\operatorname{tr} S+\operatorname{tr}_{h} \operatorname{Hess}^{\nabla} \psi-\operatorname{div}^{\nabla} \operatorname{grad}_{h} \psi+n\left|\operatorname{grad}_{h} \psi\right|_{h}^{2}-2 n a\right\} .
\end{aligned}
$$

By the pre-normalized condition and (A.2), we have

$$
a=\frac{1}{2}\left|\operatorname{grad}_{h} \psi\right|_{h}^{2},
$$

and hence (A.5) by (A.14), (A.6) by (A.15). 
As a corollary, we have the following.

Proposition A.1. For pre-normalized Blaschke immersions $F, \widetilde{F}=e^{\psi} F: M \rightarrow \mathbf{R}^{n+2}$, the formula

$$
\operatorname{tr} \widetilde{S}=e^{-2 \psi}\left(\operatorname{tr} S-\triangle_{h} \psi-\frac{n-2}{2}\left|\operatorname{grad}_{h} \psi\right|_{h}^{2}\right)
$$

holds. In particular, $\widetilde{F}$ is centroaffine minimal if and only if $\psi$ satisfies

$$
\triangle_{h} \psi+\frac{n-2}{2}\left|\operatorname{grad}_{h} \psi\right|_{h}^{2}=\operatorname{tr} S
$$

In the case of surfaces, we have the following.

Corollary A.1. For any pre-normalized Blaschke immersion $F: M^{2} \rightarrow \mathbf{R}^{4}$, there exists a function $\psi$ locally defined on $M$ such that $\widetilde{F}=e^{\psi} F$ is centroaffine minimal.

Proposition A.2. The Ricci tensor fields Ric, $\widetilde{\text { Ric }}$ of the connections $\nabla, \widetilde{\nabla}$ induced by pre-normalized Blaschke immersions $F, \widetilde{F}=e^{\psi} F: M \rightarrow \mathbf{R}^{n+2}$ satisfy

$$
\begin{aligned}
\widetilde{\operatorname{Ric}}= & \operatorname{Ric}+(1-n) \operatorname{Hess}^{\nabla} \psi+\operatorname{Hess}^{*} \psi-\triangle_{h} \psi h \\
& +(n-2)\left\{d \psi \otimes d \psi-\left|\operatorname{grad}_{h} \psi\right|_{h}^{2} h\right\} .
\end{aligned}
$$

Proof. By (A.3), we have

$$
\begin{aligned}
& \widetilde{\nabla}_{X} \widetilde{\nabla}_{Y} Z \\
= & \nabla_{X} \nabla_{Y} Z+\left\{\nabla_{Y} Z \psi+(Y \psi)(Z \psi)-h(Y, Z)\left|\operatorname{grad}_{h} \psi\right|_{h}^{2}\right\} X \\
& +\{X(Z \psi)\} Y+\{X(Y \psi)\} Z \\
& +(X \psi) \nabla_{Y} Z+(Z \psi) \nabla_{X} Y+(Y \psi) \nabla_{X} Z \\
& -\left\{h\left(X, \nabla_{Y} Z\right)-(Y \psi) h(X, Z)+X h(Y, Z)\right\} \operatorname{grad}_{h} \psi \\
& -h(Y, Z) \nabla_{X} \operatorname{grad}_{h} \psi \\
& +[(Z \psi)(Y \psi) X+(Z \psi)(X \psi) Y+(X \psi)(Y \psi) Z \\
& \left.-(Z \psi) h(X, Y) \operatorname{grad}_{h} \psi\right] .
\end{aligned}
$$

Since $\nabla h$ is symmetric, $\nabla^{*}$ is of torsion free $([7, \mathrm{p} .21])$. Hence by a direct calculation, we obtain the curvature tensor field as

$$
\begin{aligned}
\widetilde{R} & X, Y) Z \\
= & R(X, Y) Z \\
& -\left\{\operatorname{Hess}^{\nabla} \psi(Y, Z)-(Y \psi)(Z \psi)+h(Y, Z)\left|\operatorname{grad}_{h} \psi\right|_{h}^{2}\right\} X \\
& +\left\{\operatorname{Hess}^{\nabla} \psi(X, Z)-(X \psi)(Z \psi)+h(X, Z)\left|\operatorname{grad}_{h} \psi\right|_{h}^{2}\right\} Y \\
& +\{h(Y, Z) X \psi-h(X, Z) Y \psi\} \operatorname{grad}_{h} \psi \\
& +h(X, Z) \nabla_{Y} \operatorname{grad}_{h} \psi-h(Y, Z) \nabla_{X} \operatorname{grad}_{h} \psi .
\end{aligned}
$$

By taking a trace with respect to $X$, we have (A.19) from (A.1) and (A.2).

\section{References}

[1] Ferapontov, E. V., Integrable systems in projective differential geometry, Kyushu J. Math. 54(2000), no. 1, 183-215.

[2] Fujioka A., Furuhata H. and Sasaki T., Projective minimality for centroaffine minimal surfaces, J. Geom. 105(2014), no. 1, 87-102.

[3] Furuhata H., Minimal centroaffine immersions of codimension two, Bull. Belg. Math. Soc. 7(2000), no. 1, 125-134.

[4] Liu H.-L., Indefinite equi-centroaffinely homogeneous surfaces with vanishing Pick-invariant in $\mathbf{R}^{4}$, Math. J. 26(1997), no. 1, $225-251$.

[5] Lopšic, A. M., On the theory of a surface of $n$ dimensions in an equicentroaffine space of $n+2$ dimensions, (Russian) Sem. Vektor. Tenzor. Analizu. 8(1950), 286-295.

[6] Nomizu K. and Sasaki T., Centroaffine immersions of codimension two and projective hypersurface theory, Nagoya Math. J. 132(1993), 63-90. 
[7] Nomizu K. and Sasaki T., Affine differential geometry. Geometry of affine immersions, Cambridge Tracts in Mathematics, 111, Cambridge University Press, Cambridge, 1994.

[8] Sasaki T., Projective differential geometry and linear homogeneous differential equations, Rokko Lectures in Math. 5. Kobe University, 1999.

[9] Sasaki T., Line congruence and transformation of projective surfaces, Kyushu J. Math. 60(2006), no. 1, 101-243.

[10] Simon, U., Schwenk-Schellschmidt, A. and Viesel, H., Introduction to the affine differential geometry of hypersurfaces, Lecture Notes of the Science University of Tokyo, Science University of Tokyo, Tokyo, 1991.

[11] Walter, R., Centroaffine differential geometry: submanifolds of codimension 2, Results Math. 13(1988), no. 3-4, 386-402.

[12] Yang Y. and Liu H.-L., Minimal centroaffine immersions of codimension two, Results Math. 52(2008), no. 3-4, 423-437.

\section{Affiliations}

ATSUSHi FUJIOKA

ADDRESS: Department of Mathematics, Kansai University, Suita 564-8680, Japan.

E-MAIL: afujioka@kansai-u.ac.jp

HiTOSHI FURUHATA

AdDress: Department of Mathematics, Hokkaido University, Sapporo 060-0810, Japan.

E-MAIL: furuhata@math.sci.hokudai.ac.jp

TAKESHI SASAKI

AdDRESS: Department of Mathematics, Kobe University, Kobe 657-8501, Japan.

E-MAIL: sasaki@math.kobe-u.ac.jp 